nucleus, but that label became concentrated around the nuclear membrane in late S-phase. No shift of label occurred during chase periods with unlabelled thymidine and later peripheral labelling represented replication of heterochromatin close to the nuclear membrane.

All this is very confusing and the basic questions remain unanswered. Does DNA replication occur solely at the nuclear membrane or over the entire nucleus? Does DNA remain fixed in the nucleus while the replication site moves along it, or does the replication site remain fixed while the DNA is displaced relative to it, or do both replication site and DNA move?

On page 32 of this issue of Nature, Huberman, Tsai and Deich at last throw some light on this picture of confusion, especially with regard to the autoradiography experiments. The two principal sources of error in previous work have been over-long pulses of labelled thymidine and variation in methods of cell synchronization. Because DNA replicates at a rate of $0.5-1.2 \mu \mathrm{m} / \mathrm{min}$, a pulse of $2 \mathrm{~min}$ and more could result in erroneous location of originally peripheral grains in a central position in the nucleus. All of the previous autoradiographic studies involved pulse times of $5 \mathrm{~min}$ or greater.

The amethopterin method of synchronization used by Comings and Kakefuda is now known to cause cellular damage in the form of abnormal nuclear transformation. Thus Tokuyasu, identifying a cell's position in S-phase according to its centriolar development, found that the cells with heavy peripheral labelling identified by Comings as early S-phase were, in fact, late S-phase.

Huberman et al. carefully avoided these pitfalls. They used short pulse times of $0.5 \mathrm{~min}$ and the much gentler synchronization method of metaphase selection, and found general labelling throughout the nucleus in early S-phase with peripheral labelling becoming predominant in late Sphase. Because it is known that heterochromatin is late replicating, and because Huberman's pictures show a preponderance of condensed chromatin at the nuclear envelope, these results suggest that euchromatin is replicated early in S-phase and is distributed throughout the nucleus, whereas heterochromatin replicates late in S-phase and is located close to the inner nuclear membrane.

Moreover, in unsynchronized cells,
Huberman et al. found no difference in the location of grains after a 0.5 min pulse, a 10 min pulse and a 0.5 min pulse followed by a $6 \mathrm{~h}$ chase and, in synchronized cells, label from a 0.5 min pulse remained unchanged in position after a $6 \mathrm{~h}$ chase. They conclude, therefore, that DNA has a stable location in the nucleus.

Finally, Huberman et al. suggest that, in the cell fractionation experiments, it is possible that newly replicated DNA may possess special features such as extensive single strandedness which may cause it to bind more protein or membrane material than bulk DNA. Indeed, careful experiments recently reported by Fakan et al. confirm that the behaviour of newly replicated DNA in $\mathrm{CsCl}$ gradient and phenol extracts is a result of its single strandedness rather than its association with membrane material. They present evidence that newly replicated DNA is not preferentially located with nuclear membranes but is found in a fraction of chromosomal deoxyribonucleoprotein very slightly lighter than bulk deoxyribonucleoprotein and probably consisting of DNA and the additional proteins involved in DNA replication.-From a Correspondent.
TUMOUR VIROLOGY

\section{Mutant Viruses}

from our Cell Biology Correspondent

THE first report of the isolation of temperature sensitive mutants of polyoma virus appeared in 1965 and, for reasons that are far from clear, attempts to isolate similar mutants of Simian Virus 40 failed for several years. In the past two years, however, three or four groups have succeeded in isolating temperature sensitive mutants of SV40 and have begun the job of characterizing them. Tegtmeyer, for example (J. Virol., 10, 591 ; 1972), has characterized three of his mutants, $t s A 7$, $t s A 28$ and $t s A 30$, which are all in one complementation group and are apparently SV40 equivalents of Fried's tsa mutant of polyoma virus, one of the most instructive mutants of this virus. The gene that is defined by Tegtmeyer's three mutants specifies a function that is required for the initiation but not the maintenance of transformation and also for the initiation of rounds of viral DNA replication during the lytic cycle of this virus.

When non-permissive mouse $3 T 3$ cells at $39^{\circ} \mathrm{C}$ are infected with any one of the three SV40 mutants, the number of cells that are transformed is some 25 -fold to 158 -fold less than the number transformed after infections at $33^{\circ} \mathrm{C}$; the function of the mutated gene is

\title{
Direct Sequencing of Phage DNA
}

IN next week's Nature New Biology (January 10) there is a set of three reports from the MRC Laboratory of Molecular Biology in Cambridge which describe how the base sequences of two fragments of the single-stranded DNA of bacteriophage $\varphi$ X174 have been analysed directly and how one of these sequences has been identified as the 5 -amino-terminal end of the spike protein cistron of the phage genome. Ziff, Sedat and Galibert, working in Sanger's laboratory, used endonuclease IV induced by phage $\mathrm{T} 4$ to digest partially $\varphi \mathrm{X} 174$ DNA and from the digestion products they isolated a fragment fortyeight nucleotides long. After this fragment was depurinated and digested with snake venom phosphodiesterase, spleen phosphodiesterase and endonuclease IV, the sequences of the oligonucleotides which comprise this fragment were determined by fingerprint analyses. These methods should prove to be of general application for sequencing DNAs.

Robertson, Barrell, Weith and Donelson have also sequenced a fragment of $\varphi \times 174$ DNA using some of the methods developed by Ziff et al. They isolated a fragment of this viral DNA by first binding ribosomes to the DNA and then digesting away all but the sequence protected by the ribosomes. This technique has, of course, been used to isolate ribosome binding sites of the RNAs of phages $R 17$ and $Q \beta$ preparatory to sequencing.

The sequence of the $\varphi \times 174$ DNA protected by ribosome binding proved, as expected, to contain a triplet ATG, equivalent to an AUG codon; moreover, if the sequence is arranged to maximize base pairing the ATG triplet occurs at the loop of a hairpin structure. As Robertson et al. point out, this and other features of the sequence are shared by the sequences of the initiation sites of the $R 17$ and $Q \beta$ phage coat protein cistrons.

All these data indicate that the fragment of $\varphi \times 174$ DNA that has been analysed is the beginning of a cistron. In the third article of the set, Air and Bridgen report the amino-terminal amino-acid sequence of the $\varphi \times 174$ spike protein specified by cistron $G$. This amino-acid sequence is precisely that predicted from the nucleotide sequence reported by Robertson et al., who have clearly isolated and analysed the first part of the $\varphi$ X174 G cistron. 